\title{
Hypoxia-induced HMMR Promotes Cell Proliferation, Migration and Immune Infiltrates in Lung Adenocarcinoma
}

Duan Lin can ( $\nabla$ duanmumuhuosan@163.com )

Kunming Medical University https://orcid.org/0000-0001-8262-7824

Jiang Xiu lin

Kunming Medical University

Tan Lin

Kunming Medical College: Kunming Medical University

Yuan Yi xiao

Kunming Medical University

Wang Juan

Kunming Medical University

Liu Qian qian

Kunming Medical University

Zou Xiao lan

Kunming Medical University

Primary research

Keywords: Cell Proliferation, Migration and Immune Infiltrates, Lung Adenocarcinoma

Posted Date: August 3rd, 2021

DOl: https://doi.org/10.21203/rs.3.rs-757552/v1

License: (9) (i) This work is licensed under a Creative Commons Attribution 4.0 International License.

Read Full License 


\section{Abstract}

\section{Background}

Hyaluronan mediated motility receptor (also known as RHAMM) is another one of few defined hyaluronan receptors, play pivotal roles in cell growth. However, the relationships between HMMR and prognosis and tumor-infiltrating lymphocytes in lung adenocarcinoma remain unclear.

\section{Methods}

HMMR expression was analyzed emoloyed the TIMER, GEPIA, UALCAN, CCLE databases, the prognosis of HMMR was analysis by prognoscan, KMplot and GEPIA databases. The GO and KEGG pathway was analysis by the DAVID and GSEA software. The correlation between the HMMR expression was analysis by the TIMER databases, the gene and protein networks was analysis by Genemania and STRING databases, the DNA methylation was analysis by the MethSurv and UALCAN databases, the gene mutation of HMMR was analysis by the cBioportal and COSMIC databases. The expression of HMMR was analysis by $\mathrm{IHC}$ and $\mathrm{qPCR}$, the function of HMMR on cell proliferation and migration was examine by the cell growth curve, clone information, transwell and wound healing assay.

\section{Results}

In this study, we find that HMMR was elevated in LUAD and it's highly expression associated with the poor prognosis and lymph node metastasis. Furthermore, the expression of HMMR was induced by hypoxia in LUAD. HMMR expression level not only positively correlation with the different immune cells, but also positively correlation with the expression of immune checkpoints related gene, for instance, CD279, CD274, CTLA4, LAG3, PDCD1LG2, TIGIT and HAVCR2. Finally, depletion of HMMR significantly represses the cell growth and migration of NSCLC. Overall, this study emphasized the significance of HMMR in cancer progression and Immune infiltration of LUAD.

\section{Conclusions}

We demonstrated HMMR was elevated in LUAD and positively relation to poor prognosis. We find the hypoxia microenvironment and DNA hypomethylation able to up-regulation of the HMMR expression. Additionally, HMMR expression was positive with the diverse immune cell and immune regulator related gene in LUAD. Finally, we found that depletion of HMMR was inhibits the cell proliferation and migration ability of NSCLC cells. These findings suggest that HMMR could be served as a biomarker for prognosis and immune infiltration in LUAD.

\section{Introduction}

Lung cancer is one of the main causes of cancer death, and It's has brought huge economic and social burden in the worldwide[1]. Lung Cancer mainly contained the small lung squamous cell carcinoma and non-small lung squamous cell carcinoma. While, the NSCLC Composed of lung adenocarcinoma and 
lung squamous cell carcinoma[1,2]. Due to the lack of effective diagnostic markers result in most of the patients with lung cancer have been found in advanced stage, missed the best treatment time $[2,3]$. Therefore, it's imperative find specificity and sensitivity biomarkers for diagnosis and treatment of lung cancer.

HMMR, named as RHAMM, called hyaluronan mediated motility receptor, its play pivotal roles in cell growth [4]. Studies have shown that HMMR was mainly expressed in the nervous system [5]and the mouse brain [6]. The gene mutation of HMMR usually lead to the neurodevelopmental defects [7]. Research studies have shown that high HMMR expression has been associated with various cancer, such as breast cancer [8], colorectal cancer[9], stomach cancer [10], endometrial cancer [11] and prostate cancer [12]. Previous study indicated that HMMR was essential for maintains the stemness of glioblastoma stem cells[13]. However, the significance and potential mechanisms of HMMR in LUAD progression and tumor-infiltrating remains bewildered.

In this finding, we show that HMMR was highly expresison in lung adenocarcinoma and it's high expression was correlated with poor prognosis, tumor stages and lymph node metastasis. furthermore, up-regulated HMMR expression was markedly positive with the the infiltration levels of B cells, CD4 + T cells, CD8 + T cells, macrophages, neutrophils, and dendritic cells in lung adenocarcinoma. Importantly, the expression of HMMR was positively with the immune related gene expression, HMMR able to influence the prognosis of lung adenocarcinoma patients partially via immune cell infiltration. We use the qRT-PCR and IHC assay show that HMMR was highly expression in NSCLC cells and lung cancer. We also found that HMMR was induced by hypoxia via HIF-1a-dependent transcriptional regulation. Finally, we show that depletion of HMMR significantly inhibits growth and migration of NSCLC cells. These observations indicate that HMMR may plays an crucial role in the progression and immune infiltration of lung adenocarcinoma.

\section{Methods}

\section{The expression, prognosis, Clinical information and Immune infiltration analysis}

We mainly using the following databases to analysis the expression, prognosis, clinical information and Immune infiltration of HMMR in cancers. The detail information of databases used in this study are as follows: Oncomine database [14], TIMER [15],UALCAN database [16], Kaplan-Meier plotter [17] ,TISIDB database [18], the Human Disease Methylation Database [19], the PrognoScan database [20],The LinkedOmics database[21]. GEPIA database [22] and cbioportal[23].

\section{Plasmids construction and cell culture}


The shRNA for HMMR was constructed employ pLKO.1 vector. The NSCLC related cells mainly purchased from The Kunming Institute of Zoology (KIZ) of Chinese Academy of Sciences (CAS). BEAS-2B and NSCLC related cells lines were cultured using the RPMI1640 medium, this medium contain the $10 \%$ fetal bovine serum (FBS) and 1\% penicillin/streptomycin. The shRNA for HMMR primer sequences are list follows: HMMR shRNA\#1: AAACAGCTGGAAGATGAAGAAGGAA, HMMR shRNA\#2: CAGCTGGAAGATGAAGAAGGAAGAA.

\section{Quantitative real-time PCR}

The qRT-PCR assay was performed as documented [24]. The primer sequences are list follows HMMR-F: ATGATGGCTAAGCAAGAAGGC, HMMR-R: T TTTCCCTTGAGACTCTTCGAGA; $\beta$-actin-F: CTTCGCGGGCGACGAT, $\beta$-actin-R: CCATAGGAATCCTTCTGACC. The expression quantification was obtained with the $2-\Delta \Delta C$ t method.

\section{The function assays of HMMR In LUAD}

The function assay of HMMR was performed as documented [25]. The function assay of HMMR mainly included the cell growth curve, colony information, transwell and wound healing experiment. The cell growth curve and colony information assay mainly employed to estimate the cell proliferation ability of NSCLC cells after the depletion of HMMR. The transwell and wound healing experiment mainly employed to estimate the cell migration ability of NSCLC cells after the depletion of HMMR.

\section{Western Blotting and Immunohistochemistry staining}

The Western Blotting and Immunohistochemistry staining assay was performed as documented [29].Briefly, Cell lysates were collected, perform the Western blot, primary antibody overnight incubation and second antibody incubation. Finally, develop using instrument.The detail information of antibodies employ in our study are as follows:HMMR antibody (CST Group (HMMR, Rabbit mAb \#87129, 1:1000) and $\beta$-actin (Catalog number: 66009-1-lg , 1:20000, Proteinch, Shanghai, China).

\section{Statistical Analysis}

The significance of the data between two assays groups was decided by Student's t-test, $\mathrm{P}<0.05\left(^{*}\right), \mathrm{P}<$ $\left.0.01{ }^{(\star \star}\right)$ and $\left.\mathrm{P}<0.001{ }^{(\star \star \star}\right)$, was considered significantly.

\section{Results}

\section{The mRNA of HMMR was elevated in human cancer}


In order to examine the mRNA of HMMR expression pattern in multifarious cancer, we employed the TIMER tools to analysis the expression of HMMR, the find shown that HMMR was elevated in as follow cancer than the control group, it mainly includes BLCA, BRCA, CHOL, COAD, ESCA, HNSC, KIRC, KIRP, LIHC, LUAD, LUSC, PRAD, READ, STAD, THCA and UCEC (Figure 1A). To further verify the results, we using the combine the TCGA and GTE databases to figure out the HMMR expression. As is show in Figure 1B, the HMMR was significantly up-regulation in ACC, BLCA, BRCA, CESC, COAD, DLBC, ESCA, GBM, HNSC, LIHC, LUAD, LUSC, OV, PAAD, READ, SKCM, STAD, THYM, UCEC and UVM cancer than match healthy tissue, while low expression in LAML and TGCT(Figure 1B). Besides, we found that the HMMR was highly expression in NSCLC cells lines observe in CCLE network tools (Figure 1C). Above all, our findings indicated that the HMMR may play an crucial roles in the progression of cancers.

\section{The prognostic value of HMMR in pan-cancers}

Then, through analysis the TCGA databases, we find the expression of HMMR has the great significance for assessment the prognostic value of different cancers. High expression of HMMR was associated with poor OS in ACC, COAD, KICH, KIRC, KIRP, LGG, LIHC), LUAD, MESO, PAAD, PCPG, PRAD, THYM and UVM (Figure 2A), and related to poor DSS in KIRP, LIHC, MESO, SARC and THCA (Figure 2B), and related to poor DFS in ACC, KICH, KIRC, KIRP, LGG, LIHC, LUAD, MESO, PRAD, THCA and UVM (Figure 2C), and linkage to poor PFS in ACC, KICH, KIRC, KIRP), LGG, LIHC, LUAD, MESO, PCPG and UVM (Figure 2D).

\section{Analysis the pathological stage in different cancer}

We employ the GEPIA tools to examine the relationship between the expression of HMMR and the human cancers pathological stage. Interestingly, we find that the expression of HMMR was markedly positive with the pathological stage of ACC, BRCA, ESCA, NHCS, KICH, KIRC, KIRP, LAML, LAML, LIHC, LUAD and LUSC (Figures 3). These results suggested that HMMR may as an oncogene in human cancers.

\section{The expression pattern of HMMR in Immune and Molecular Subtypes of cancers}

Previous reports shown that the cancer could accord to the molecular characteristics divided into different immune and molecular subtypes. Thus, we adopt the TISIDB tools to analysis the expression of HMMR in immune and molecular Subtypes of different human cancer. Concerning the immune subtypes, the analysis results shown that HMMR has a differential expression pattern in cancers (Figure 4A), a quintessential example should be cited LUAD, the HMMR was elevated in $\mathrm{C} 1$ and $\mathrm{C} 2$, while, it's low expression was observe in $\mathrm{C} 3$. With regard to the molecular subtypes, HMMR also display a distinctive expression pattern (Figure 4B), such as, the HMMR was highly expression in C1 of LUAD, decreased in C3. To summarize, our result indicated that the expression pattern of HMMR has the tissue dependence specificity. 


\section{HMMR was highly expression in LUAD}

Considering the significance of HMMR in cancers, next, we want to explore the relationship between the expression of HMMR and clinical features in LUAD. First, we find that the RNA and protein of HMMR was significantly elevated in LUAD by perform the UALCAN tools analysis (Figure 5A-5B). In addition, we also find the expression of HMMR was increased with the elevation of stage nodal metastasis (Figure $5 \mathrm{C}$ ). The expression of HMMR not only highly expression in Ttp53-mutant patients but also upregulated in long term smoking patients. For the gender, the expression of HMMR significantly elevated in male patients than female (Figure 5D-5F). Surprisingly, we find higher expression of HMMR has the poor overall survival, poor OS, PFS and PPS (Figure 5G-5I). Finally, we also evaluate the connection of among the expression of HMMR and MKI67 (famous cell proliferation index), the result suggested that HMMR expression was markedly positive with the expression of mki67 (Figure 5K). These findings strongly imply that the HMMR may involve in the progression of LUAD.

\section{The gene mutation of HMME analysis}

For explore the gene mutation information about the HMMR, we employ the cBioportal tools preform comprehensive analysis regard to the HMMR. The result shown that the mutation rate of HMMR reached $2.9 \%$ in NSCLC (Figure 6A), we also examine the mutation type and base mutation in NSCLC, we found that Missense substitution and base $G>A$ reached the highest mutation rate in NSCLC (Figure 6B-6C). The results also display the mutation of HMMR in different NSCLC molecular Subtypes (Figure 6D), In DNA level, gain and diploid was the main reason for the HMMR high expression in NSCLC (Figure 6E). Overall, these results emphasize the gene mutation of HMME may be contribute to the HMMR elevated in NSCLC.

\section{Analysis DNA methylation of HMMR in LUAD}

Next, we further explore the connection of among the DNA methylation of and the expression of HMMR, as is shown in the (Figure 7A), we display varies methylation sites locate in the promoter of HMMR by heat map. Through preform the comprehensive and detailed analysis about the DNA methylation state of HMMR, we find the methylation level was significantly low expression in the LUAD, as well as the methylation level was gradually decreased with the elevation of pathological stage in LUAD (Figure 7B7C). The methylation level was gradually decreased observe in the different lymph node metastasis state, race and tp53mutation (Figure 7D-7G). The overall survival analysis display that elevated the methylation level of HMMR has better prognosis (Figure 7H). These finds suggested that DNA hypomethylation may be reason for the abnormal up-regulation in LUAD.

\section{Analysis The function of HMMR in LUAD}


In order to exploration the potential significance of HMMR in LUAD progression. We first employ the linkedomics database to examine the positive gene with HMMR. As is show in the Figures $8 \mathrm{~A}-8 \mathrm{C}$, we choose the most positive gene( $r>0.7)$ display in the form of heat map. Next, we perform the GO and KEGG analysis. The biology processes mainly involve in DNA replication, chromosome segregation, cell division and protein localization (Figures 8D), for KEGG enriched results, the pathway mainly including cell cycle, P53 signaling pathway, non-small cell lung cancer and FOXO signal pathways (Figures 8E). In addition, we analysis the most relevant gene of HMMR by employed the Genemania, the result indicated that $x \mathrm{x}$ gene were most relevant, these gene functions mainly involve in cell cycle (Figure 8F). We also using STRING databases to construct the protein interaction network, the protein of interaction with HMMR mainly including PLK4, CD44, AURKA, NEK2, CDK1, FAM83D(Figure 8G). To explore the HMMR related signal pathway in progression of LUAD, we employ the GSEA software perform KEGG pathway enrich. The analysis results show that upregulation HMMR expression mainly involve in the IL2-STAT5 signaling pathway, IL6 JAK-STAT3- signaling pathway, Interferon- $\gamma$ response, TNFa signaling pathway(Figure 8H). These results suggested that HMMR plays an pivotal roles in the immune response regulation in LAUD.

\section{Hypoxia induced the HMMR highly expression in LUAD}

The GSEA enrich results show that HMMR mainly participate in hypoxia process (Figure 9A), we guess the HMMR expression whether by hypoxia induced. To test this hypothesis, we employ the JASPAR and AnimalTFDB tools to predict the HIF1a HRE in the promoter of HMMR. Next, we analysis the relationship among HMMR expression and HIF1a expression in TCGA LUAD, the result indicated that HIF1a significantly positive with the expression of HMMR (Figure 9B). In addition, we using the different condition to treat the NSCLC cells, such as hypoxia and cocl2, cocl2 as an chemical inducer of HIF1a, through these condition treat, compared with normoxia, we find the hypoxia and cocl 2 was able to increase the expression of HMMR (Figure 9C). We further analysis the relationship among HMMR expression and HIF1a downstream targets gene expression (eg囚LDHA, PGK1, SLC2A1, GBE1) in TCGA LUAD, the results suggested that HMMR expression was strongly positive with these gene (Figure 9D9G). Beside, we find that the RNA of HIF1a was significantly elevated in LUAD by perform the UALCAN tools analysis (Figure 9H). We also find the expression of HIF1a was increased with the elevation of pathological stage and lymph node metastasis (Figure 9I-9J). Surprisingly, we find higher expression of HIF1a has the poor overall survival (Figure 9K). These findings strongly imply that the HMMR expression may be induced by the hypoxia.

\section{Analysis the correlation between the expression of HMMR and Immune infiltration in LUAD}

We employ the TIMER databases analysis examine the relationship between the expression of HMMR and Immune infiltration in LUAD, the results show that gene copy number change of HMMR was significantly affect the immune infiltration level of B cells, CD4+ T cells, CD8+ T cells, Macrophage cells, 
dendritic cells and neutrophils in LUAD (Figure 10A). Next, we used TIMER to analyze the relationship between the HMMR level and immune infiltration levels in LUAD. The results showed that HMMR expression is markedly positively associated with $B$ cells $(r=0.47, p=7.69 e-30), C D 4+T$ cells $(r=0.71$, $p=1.71 e-78), C D 8+T$ cells $(r=0.51, p=1.22 e-35)$, Macrophage cells $(r=0.44, p=8.88 e-26)$, dendritic cells $(r=0.84, p=5.94 e-139)$ and neutrophils $(r=0.77, p=3.54 e-103)$ in LUAD (Figure 10B). To evaluate the relationship among HMMR expression and immune checkpoints related gene, for instance: CD274, CD279, CTLA4, LAG3, PDCD1LG2, TIGIT and HAVCR2. The results show that HMMR expression was memorably positively relation to $C D 274(r=0.34, P=9.3 e-15), C T L A 4(r=0.556, P=5)$, PDCD1 $(r=0.56, P=0)$, TIGIT $(r=0.71, P=0), P D C D 1 L G 2(r=0.67, P=0)$, HAVCR2 $(r=0.71, P=0)$ and LAG3 $(r=0.39, P=0)$ in LUAD (Figure 10C). These results memorably indicated that HMMR play an crucial roles in tumor immune infiltration regulator in LUAD.

\section{The prognostic of HMMR based on the different immune cells}

To explore the prognostic of HMMR based on the different immune cells in LUAD. We using the KMplot database analysis found that elevated the HMMR expression as well as enriched the B cells, CD4+ T-cells, CD8+ T cells, macrophages, natural killer T cells, regulatory $T$ cells cohort had a poor prognosis (Figures $11 \mathrm{~A}-\mathrm{L})$. These data indicated that the immune cell infiltration could significantly affect the e prognostic of HMMR in LUAD.

\section{Depletion of HMMR inhibits the cell proliferation and migration of NSCLC cells}

To further definite the function of HMMR in NSCLC progression, we first using the IHC and qRT-PCR assays examine the expression of HMMR in different NSCLC cells lines, the results show that HMMR significantly elevated in the lung cancers and NSCLC cells (Figures 12A-12B), especially in A549 and $\mathrm{H} 1299$ cells. Next, we construction the HMMR knockdown cell lines in A549 and H1299 cells as well as employ the qRT-PCR and Western blot examine the knock down efficiency(Figures 12C-12D), through the loss of functions shown that depletion of HMMR inhibits the cell growth and migration of NSCLC cells(Figures 12E-12H). These findings display that HMMR boost the cell growth and migration of NSCLC cells.

\section{Discussion}

Normal hypoxic environments play a key role in pathologic and physiologic conditions [26]. Previous study have indicated that hypoxia can induce the expression of varies oncogene and promote the cancer development [26]. Hypoxia was necessary for the self-renewal of cancer stem cells [27]. In addition, numerous results have shown that HIF-1a can also directly upregualtion the expression of VEGFR1 and 
activation cancer-related signaling pathway, result in the progression of cancers. While, the expression of HMMR whether induced by hypoxia need to be further explored.

HMMR was report plays crucial roles in the progression of human cancer [13] . For example, studies have shown that HMMR was elevated in gbm, high expression of HMMR could boost the self-renewal of GSC [13]. In breast cancer, elevated the levels of HMMR are related to poor outcome [28]. At the present time, important evidence is still lacking about the significance of HMMR in the progression of LUAD. We also adopt STRING databases to construct the protein interaction network, the protein of interaction with HMMR mainly including PLK4, CD44, AURKA, NEK2, CDK1 and FAM83D. It has been show that PLK4 was elevated in NSCLC and high expression was related to the tumor size and lymph node metastasis [29]. Recent one study found that CD44 able to elevated the expression of PDL1 by regulation the CD274 transcription, result in restrain the tumor-intrinsic function of PD-L1 [30]. It has been report that the alisertib, as an inhibitor of AURKA, combined with PD-L1 blockade able to employ treat the mammary tumors [31]. NEK2 was report that elevated in lung cancer, regulated by EGFR mutation, over-expression of NEK2significantly promotes the cell growth and induce the cell cycle progression in NSCLC cells [32]. The above results indicate that HMMR may plays a central regulate role in the cancer progression.

In this study, we employ varies bioinformatics database conduct a comprehensive and detailed analysis about the expression, prognosis, clinical significance, Immune infiltration and biological function in LUAD. We find the HMMR was highly expression in various cancer tissues, such as ACC, BRCA, KIRC, KIRP, LIHC, LUAD, LUSC, OV, SKCM, TGCT, THCA and THYM Øhigh expression of HMMR was significantly correlated with the poor prognosis, tumor stages and lymph node metastasis of LUAD.

Hypoxia microenvironment and epigenetic modication play an crucial role in control gene expression [33]. Therefore, Hypoxia microenvironment and DNA hypomethylation may be a cause for HMMR elevated in LUAD. Here, we first revealed that elevated the HMMR expression was relation to the immune infiltration of $B$ cells, CD4+ T cells, CD8+ T cells, neutrophils, macrophages, and dendritic cells. Moreover, HMMR expression was also positively relation to various immune check points related gene was observed in lung cancer. Interestingly, HMMR affect the existence state of LUAD patients partially via immune cell infiltration. Our GSEA enrichment analysis results showed that HMMR is closely associated with cell immune-related pathways such as interferon $y$ response, TNF a signaling pathway, IL6-JAK-STAT3 signaling pathway and IL2-STAT5 signaling pathway. These findings suggested that HMMR could be as a immune-related biomarker in LUAD. Finally, we find that knockdown of HMMR significantly reduced the ability of proliferation and migration of NSCLC cells. These results demonstrate that HMMR promote the cell growth and migration of NSCLC cells.

In our study, we demonstrated HMMR was elevated in LUAD and positively relation to poor prognosis. We find the hypoxia microenvironment and DNA hypomethylation able to up-regulation of the HMMR expression. Moreover, our finding also suggested that HMMR promote the LUAD progression by boosts the ability of cell proliferation and migration in LUAD. 


\section{Declarations}

\section{Data Availability}

The original data was display in this study are included in the article.

\section{Funding}

This work was supported by Yunnan Applied Basic Research Projects (YNWRMY-2019-067, 2019FE001) and Yunnan Province Specialized Training Grant for High-Level Healthcare Professionals (D201614)

\section{Conflict of Interest}

The authors declare no competing interests.

\section{Ethics Statement}

The studies involving human participants were reviewed and approved by Ethics Committee of The Third Affiliated Hospital of Kunming Medical University.

\section{Acknowledgments}

The authors would like to thank support from department of Department of Thoracic Surgery II, The Third Affiliated Hospital of Kunming Medical University (Yunnan Tumor Hospital), Kunming, China.

\section{Author information}

\section{Affiliations}

Department of Thoracic Surgery II, The Third Affiliated Hospital of Kunming Medical University (Yunnan Tumor Hospital), Kunming, China.Xiulin Jiang, Lin Tang, Yixiao Yuan, Juan Wang, Qianqian Liu, Xiaolan Zou and Lincan Duan

\section{Author Contributions}

X.L-J, L.T and Y.X-Y designed this work, performed related assay and analyzed data. J.W., Q.Q-L and X.L-Z contributed to study materials, L.C-D supervised and wrote the manuscript. All authors have read and 
approved the final version of the manuscript.

\section{Ethics declarations}

Ethics approval and consent to participate

Not applicable.

\section{Consent for publication}

Not applicable.

\section{Competing interests}

The authors declare that they have no competing interests.

\section{References}

1. Bade, B.C. and C.S. Dela Cruz, Lung Cancer 2020: Epidemiology, Etiology, and Prevention. Clin Chest Med, 2020. 41(1): p. 1-24.

2. Zheng, M., Classification and Pathology of Lung Cancer. Surg Oncol Clin N Am, 2016. 25(3): p. 44768.

3. Zeng, Y., et al., Prognostic Value and Related Regulatory Networks of MRPL 15 in Non-Small-Cell Lung Cancer. Front Oncol, 2021. 11: p. 656172.

4. He, Z., et al., Hyaluronan Mediated Motility Receptor (HMMR) Encodes an Evolutionarily Conserved Homeostasis, Mitosis, and Meiosis Regulator Rather than a Hyaluronan Receptor. Cells, 2020. 9(4).

5. Casini, P., I. Nardi, and M. Ori, RHAMM mRNA expression in proliferating and migrating cells of the developing central nervous system. Gene Expr Patterns, 2010. 10(2-3): p. 93-7.

6. Lindwall, C., et al., Selective expression of hyaluronan and receptor for hyaluronan mediated motility (Rhamm) in the adult mouse subventricular zone and rostral migratory stream and in ischemic cortex. Brain Res, 2013. 1503: p. 62-77.

7. Prager, A., et al., hmmr mediates anterior neural tube closure and morphogenesis in the frog Xenopus. Dev Biol, 2017. 430(1): p. 188-201.

8. Assmann, V., et al., The pattern of expression of the microtubule-binding protein RHAMM/IHABP in mammary carcinoma suggests a role in the invasive behaviour of tumour cells. J Pathol, 2001. 195(2): p. 191-6.

9. Zlobec, I., et al., RHAMM, p21 combined phenotype identifies microsatellite instability-high colorectal cancers with a highly adverse prognosis. Clin Cancer Res, 2008. 14(12): p. 3798-806. 
10. Li, $\mathrm{H}$., et al., Expression of hyaluronan receptors CD44 and RHAMM in stomach cancers: relevance with tumor progression. Int J Oncol, 2000. 17(5): p. 927-32.

11. Rein, D.T., et al., Expression of the hyaluronan receptor RHAMM in endometrial carcinomas suggests a role in tumour progression and metastasis. J Cancer Res Clin Oncol, 2003. 129(3): p. 161-4.

12. Gust, K.M., et al., RHAMM (CD168) is overexpressed at the protein level and may constitute an immunogenic antigen in advanced prostate cancer disease. Neoplasia, 2009. 11(9): p. 956-63.

13. Tilghman, J., et al., HMMR maintains the stemness and tumorigenicity of glioblastoma stem-like cells. Cancer Res, 2014. 74(11): p. 3168-79.

14. Rhodes, D.R., et al., ONCOMINE: a cancer microarray database and integrated data-mining platform. Neoplasia, 2004. 6(1): p. 1-6.

15. Li, T., et al., TIMER: A Web Server for Comprehensive Analysis of Tumor-Infiltrating Immune Cells. Cancer Res, 2017. 77(21): p. e108-e110.

16. Chandrashekar, D.S., et al., UALCAN: A Portal for Facilitating Tumor Subgroup Gene Expression and Survival Analyses. Neoplasia, 2017. 19(8): p. 649-658.

17. Nagy, Á., G. Munkácsy, and B. Győrffy, Pancancer survival analysis of cancer hallmark genes. Sci Rep, 2021. 11(1): p. 6047.

18. Ru, B., et al., TISIDB: an integrated repository portal for tumor-immune system interactions. Bioinformatics, 2019. 35(20): p. 4200-4202.

19. Lv, J., et al., DiseaseMeth: a human disease methylation database. Nucleic Acids Res, 2012. 40(Database issue): p. D1030-5.

20. Mizuno, H., et al., PrognoScan: a new database for meta-analysis of the prognostic value of genes. BMC Med Genomics, 2009. 2: p. 18.

21. Vasaikar, S.V., et al., LinkedOmics: analyzing multi-omics data within and across 32 cancer types. Nucleic Acids Res, 2018. 46(D1): p. D956-d963.

22. Tang, Z., et al., GEPIA: a web server for cancer and normal gene expression profiling and interactive analyses. Nucleic Acids Res, 2017. 45(W1): p. W98-w102.

23. Cerami, E., et al., The cBio cancer genomics portal: an open platform for exploring multidimensional cancer genomics data. Cancer Discov, 2012. 2(5): p. 401-4.

24. Jiang, L.P., et al., GRK5 functions as an oncogenic factor in non-small-cell lung cancer. Cell Death Dis, 2018. 9(3): p. 295.

25. Duan, L., et al., Inhibitory effect of Disulfiram/copper complex on non-small cell lung cancer cells. Biochem Biophys Res Commun, 2014. 446(4): p. 1010-6.

26. Xiong, Q., et al., Hypoxia and cancer related pathology. Cancer Lett, 2020. 486: p. 1-7.

27. Soeda, A., et al., Hypoxia promotes expansion of the CD133-positive glioma stem cells through activation of HIF-1alpha. Oncogene, 2009. 28(45): p. 3949-59.

28. Wang, C., et al., The overexpression of RHAMM, a hyaluronan-binding protein that regulates ras signaling, correlates with overexpression of mitogen-activated protein kinase and is a significant 
parameter in breast cancer progression. Clin Cancer Res, 1998. 4(3): p. 567-76.

29. Zhou, Q., G. Fan, and Y. Dong, Polo-like kinase 4 correlates with greater tumor size, lymph node metastasis and confers poor survival in non-small cell lung cancer. J Clin Lab Anal, 2020. 34(4): p. e23152.

30. Kong, T., et al., CD44 Promotes PD-L 1 Expression and Its Tumor-Intrinsic Function in Breast and Lung Cancers. Cancer Res, 2020. 80(3): p. 444-457.

31. Yin, T., et al., Aurora A Inhibition Eliminates Myeloid Cell-Mediated Immunosuppression and Enhances the Efficacy of Anti-PD-L1 Therapy in Breast Cancer. Cancer Res, 2019. 79(13): p. 34313444.

32. Chen, C., et al., High expression of NEK2 promotes lung cancer progression and drug resistance and is regulated by mutant EGFR. Mol Cell Biochem, 2020. 475(1-2): p. 15-25.

33. Zhou, F., et al., CTHRC1 Is a Prognostic Biomarker and Correlated With Immune Infiltrates in Kidney Renal Papillary Cell Carcinoma and Kidney Renal Clear Cell Carcinoma. Front Oncol, 2020. 10: p. 570819.

\section{Figures}



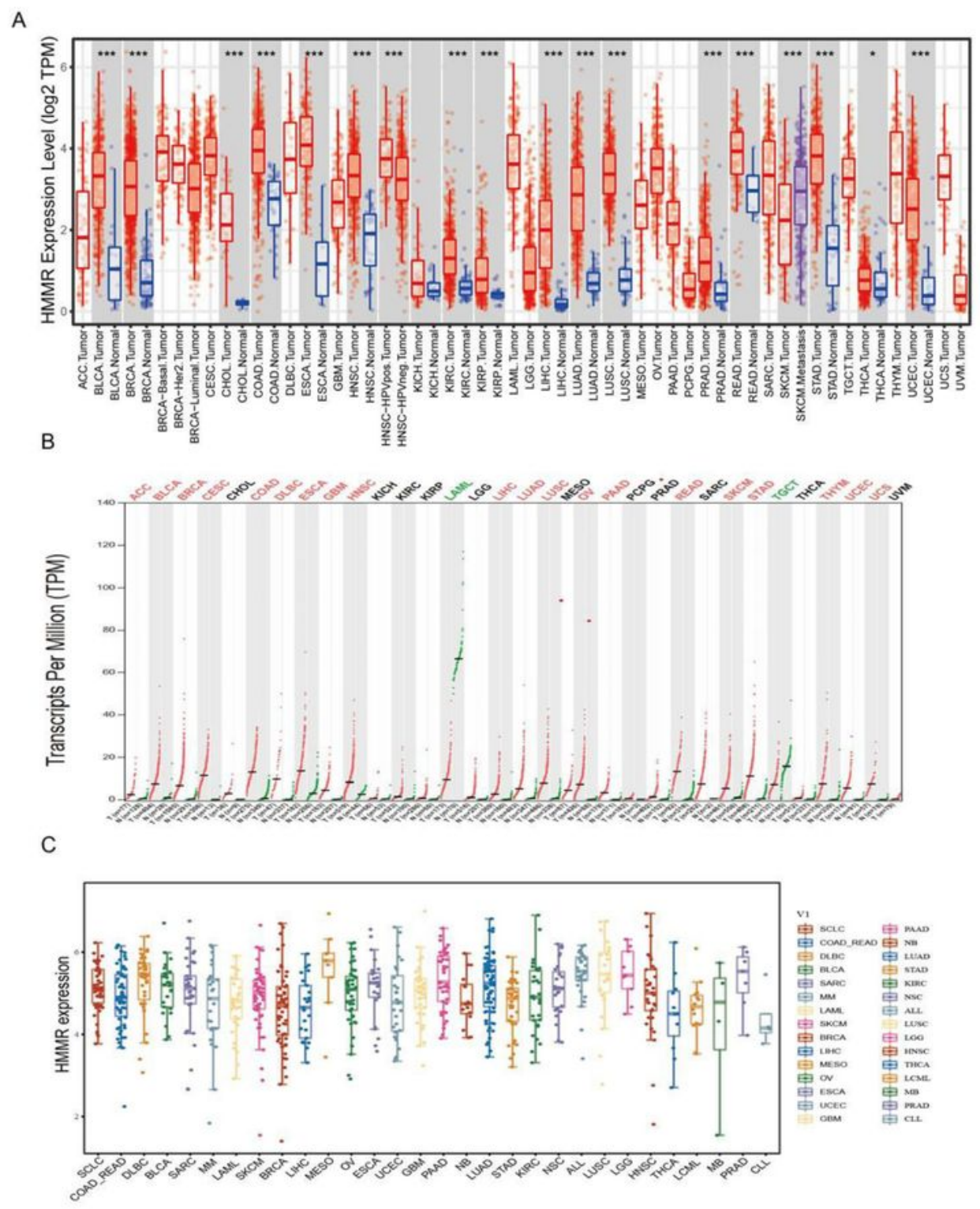

\section{Figure 1}

Analysis of the HMMR expression level in human cancers (a) Analysis the HMMR expression in pancancers by employed the TIMER tools (b) Analysis the HMMR expression in pan-cancers by employed the GEPIA tools (c) Analysis the HMMR expression in tumor cells lines by employed the CCLE database. 
A

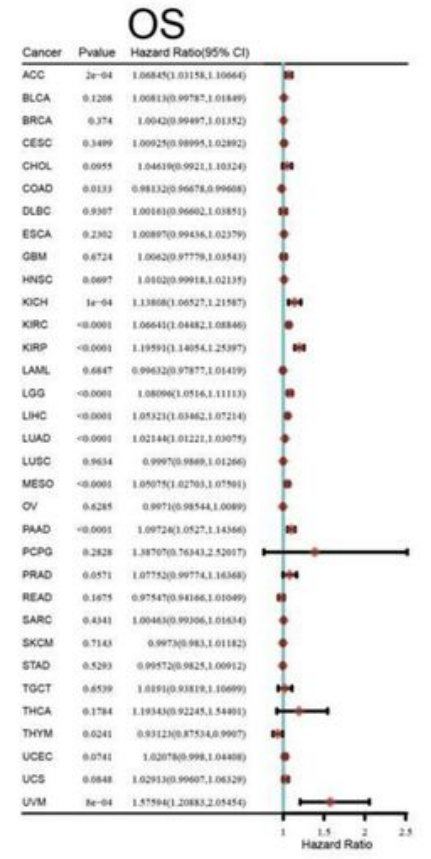

C

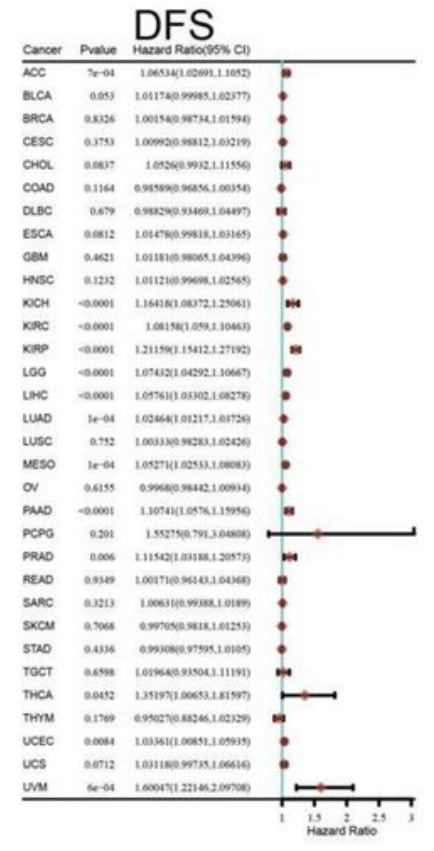

B

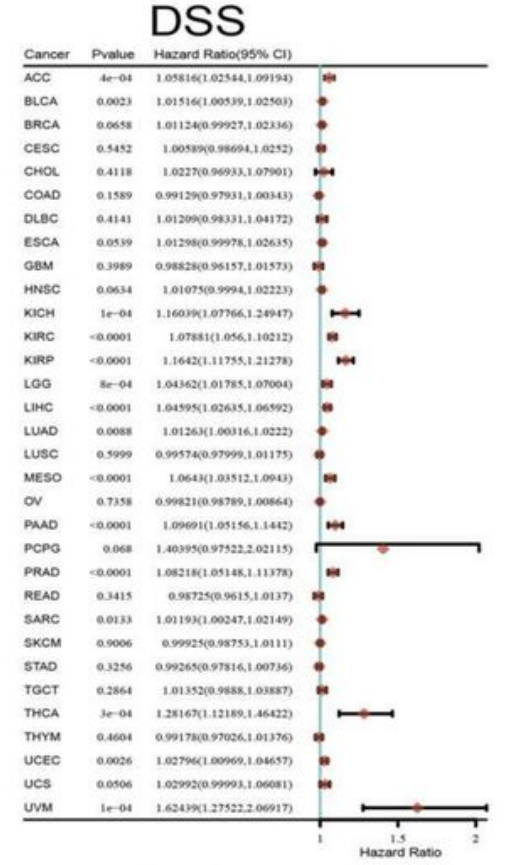

D

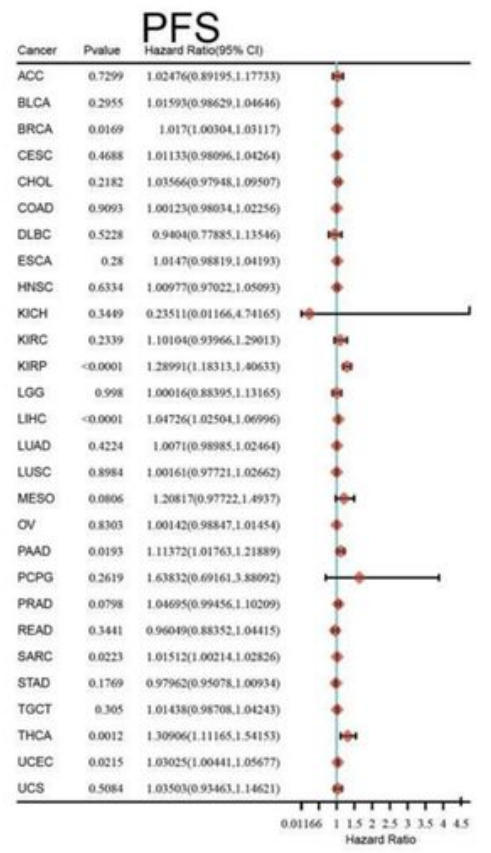

\section{Figure 2}

Analysis the prognosis of HMMR expression in Multiple cancers (a-d) Analysis the OS (A), DSS (B), DFS (C), PFS (D) of HMMR in Pan-cancers. 

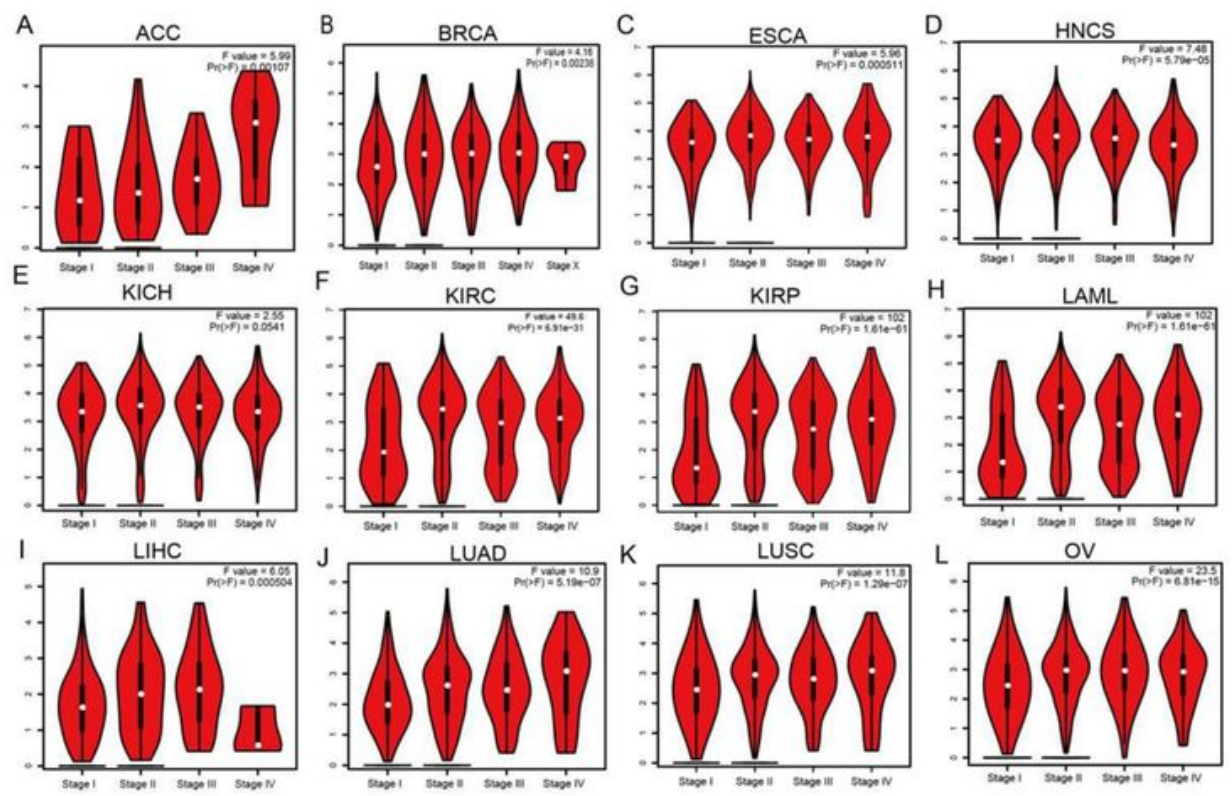

Figure 3

The pathological stage analysis for HMMR in various human cancers $(a-k)$ The pathological stage of HMMR in ACC (A), BRCA (B), ESCA(C), HNSC (D), KICH(E), KIRC(F), KIRP(G), LAML(H), LIHC(I), LUAD (J), LUSC(K) and OV(L) 
A
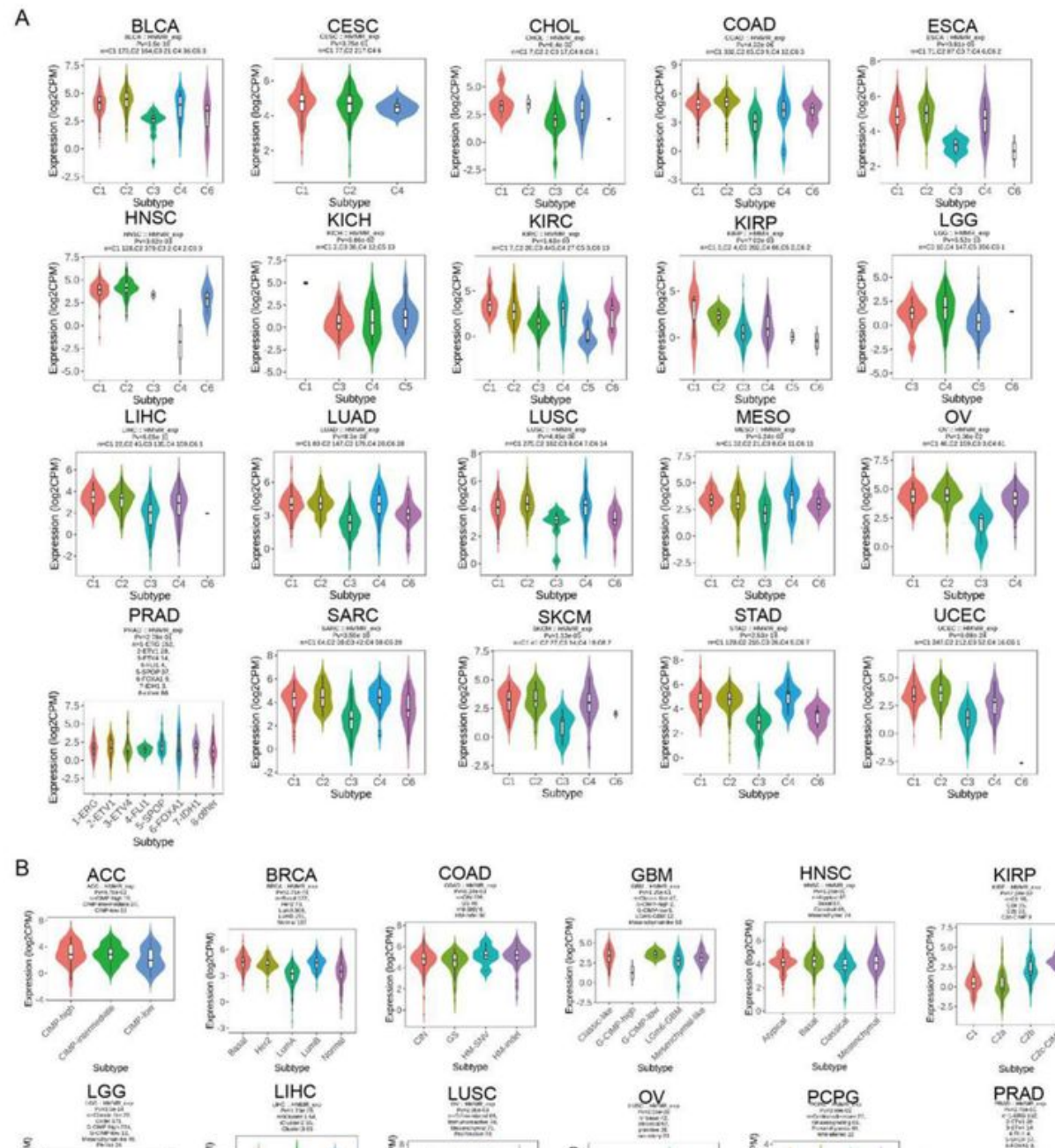

SIst
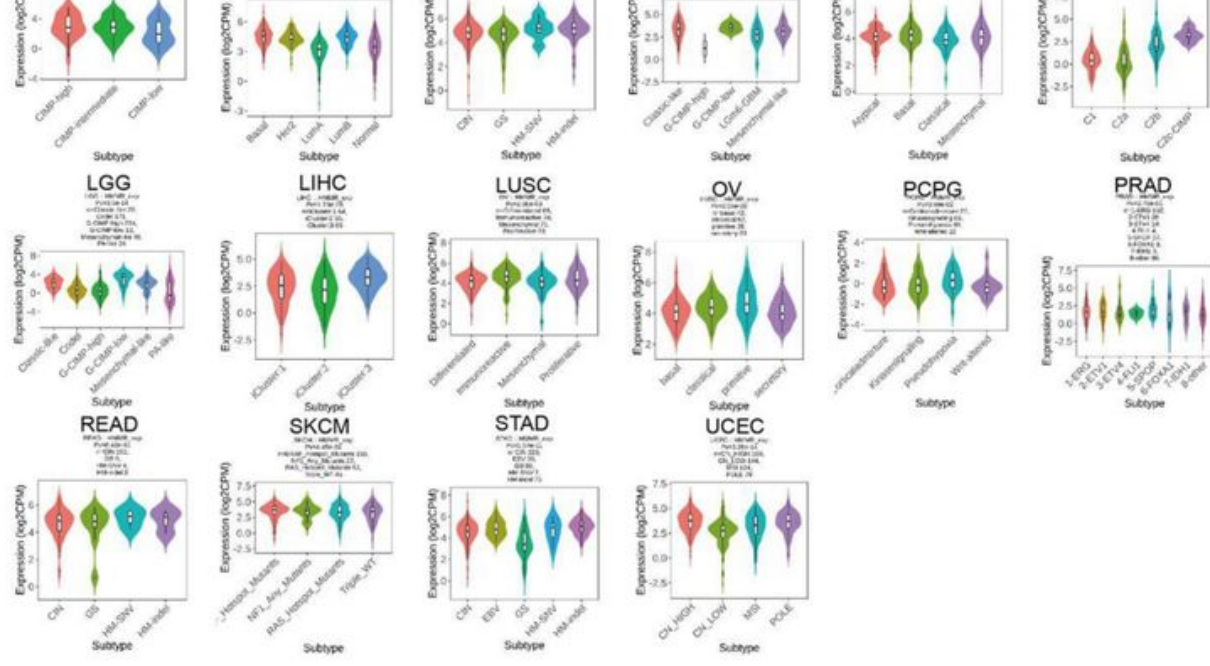

\section{Figure 4}

HMMR Expression was associated with Immune and Molecular Subtypes in Human Cancers (a) The expression of HMMR Expression in different Immune Subtypes of Multiple cancers (b) The expression of HMMR Expression in different Molecular Subtypes of Multiple cancers 
A

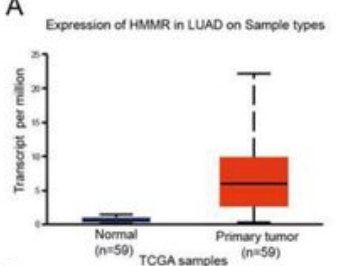

D

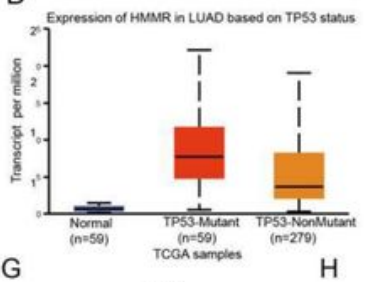

B

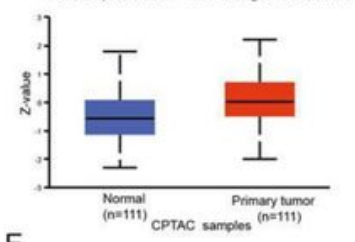

E

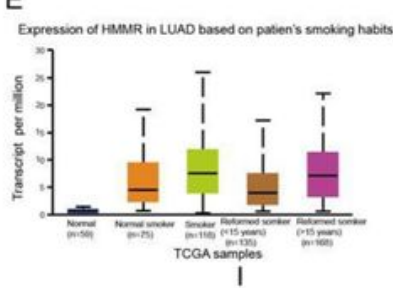

C

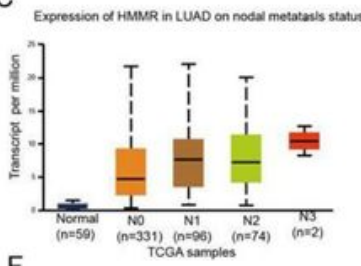

$\mathrm{F}$
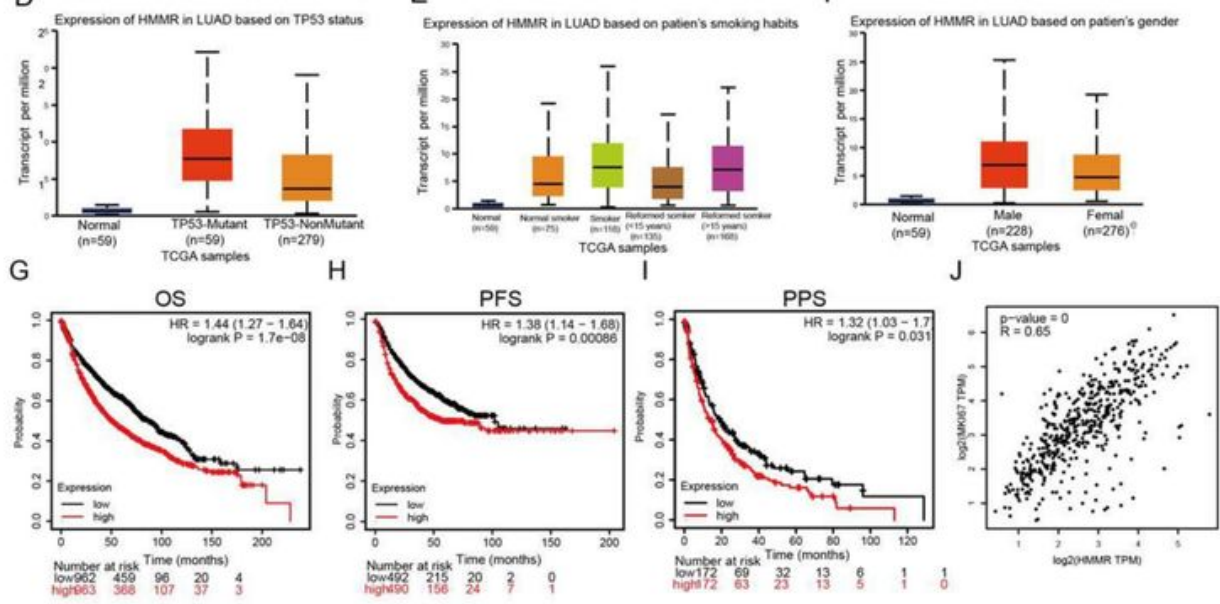

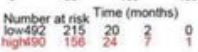

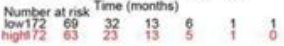

\section{Figure 5}

HMMR is elevated in LUAD (a) The mRNA of HMMR in TCGA LUAD by UALCAN databases (b) The protein of HMMR in TCGA LUAD by UALCAN databases (c) The lymph node metastatic stage analysis for HMMR in LUAD determined by GEPIA database (d) HMMR expression in group of TP53 mutation by UALCAN database (e) HMMR expression in group of smoking habits by UALCAN database (f) HMMR expression in 
group of sexs by UALCAN database ( $\mathrm{g}-\mathrm{i})$ The OS (G), PFS (H) and PPS (I) of HMMR in LUAD by KMplot database (j) The relationship of Between the HMMR expression and MKI67 expression in TCGA-LUAD

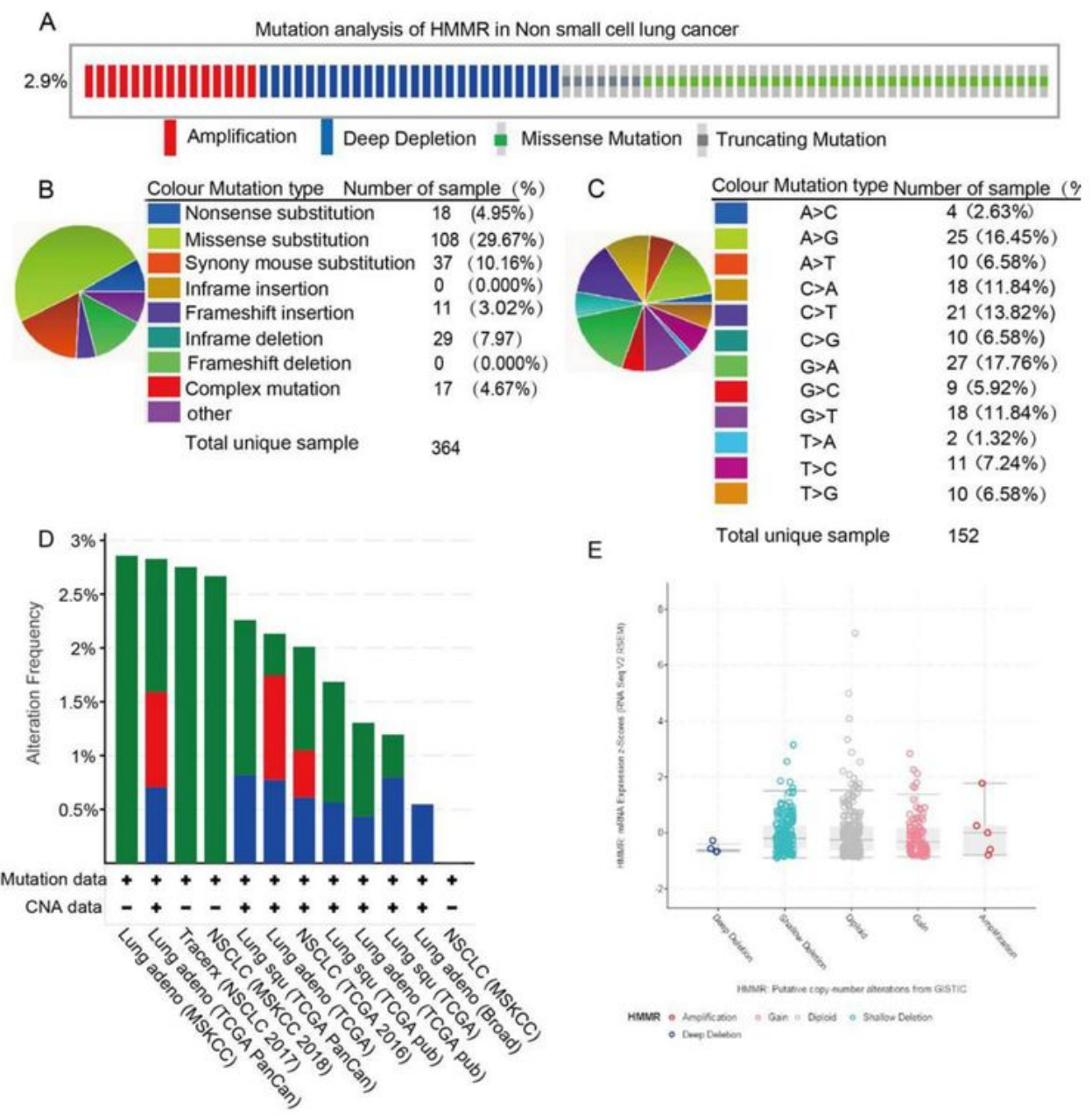

Figure 6

Mutation, Copy Number Variation Analysis of HMMR (a)The gene mutations of HMMR in lung cancer adopt the cBioportal tools (b-c) The Somatic Mutations HMMR in lung cancer adopted the Cancer 
database. (d) HMMR is frequently amplified in lung cancers. (e) HMMR expression in different CNV groups.

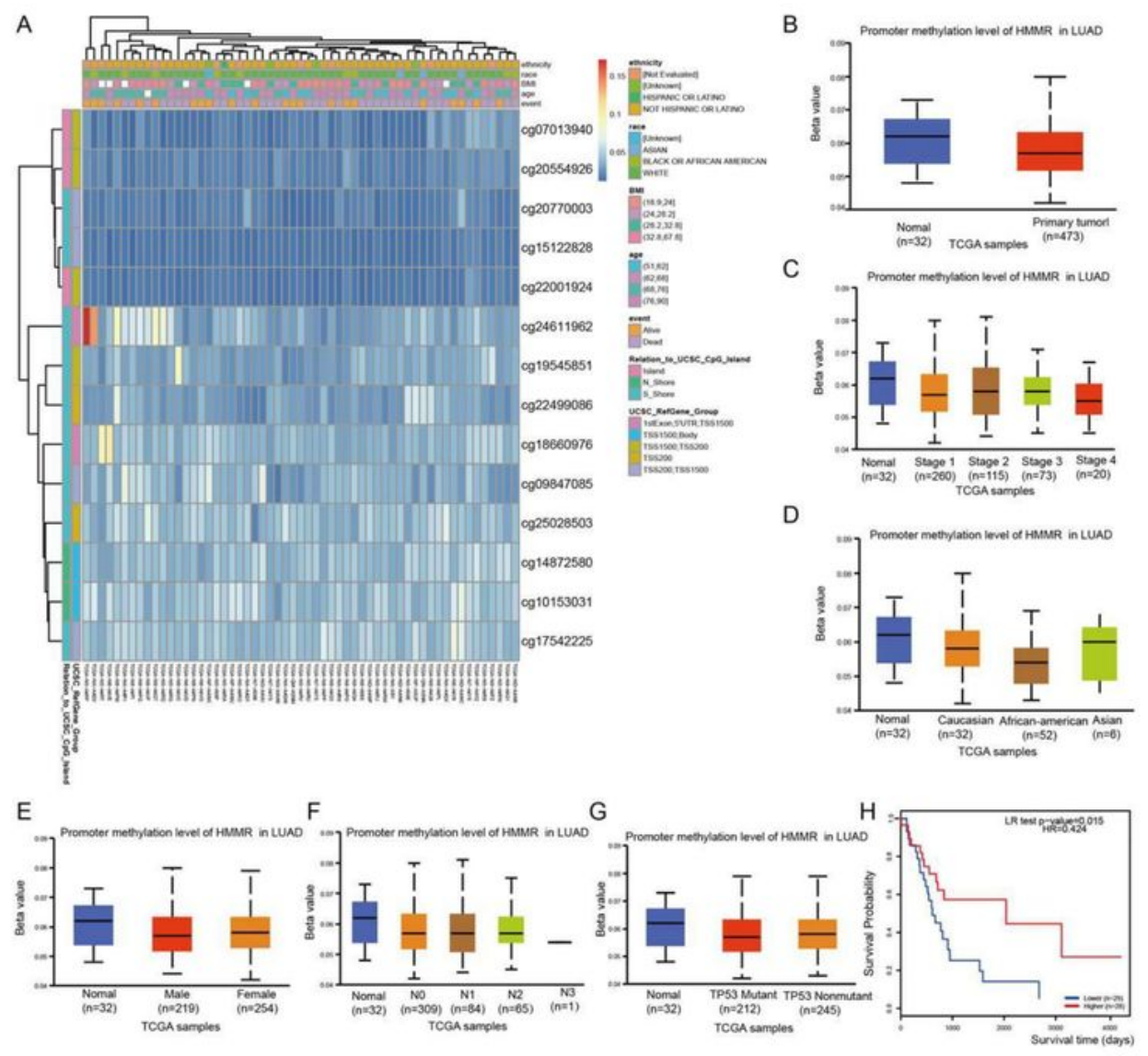

\section{Figure 7}

Analysis the DNA Methylation of HMMR $₫ a \unrhd T h e$ differential methylation regions related to HMMR were presented as heatmaps. $₫ \mathrm{~b} \otimes T$ The methylation of HMMR in LUAD. $\triangle \mathrm{C} \bigotimes$ The methylation of HMMR in different stage in the TCGA dataset. $₫ \mathrm{~d} \otimes T$ The methylation level of HMMR in different ethnicity in the TCGA dataset. 
\e\The methylation level of HMMR in different group of sex in the TCGA dataset. $₫ \mathrm{f} \otimes T$ The methylation level of HMMR in different group of metastasis status in the TCGA dataset. $₫ \mathrm{~g} \unrhd$ The methylation level of HMMR in different group of TP53 type in the TCGA dataset. खh囚The OS of the methylation level of HMMR in the TCGA LUAD dataset.

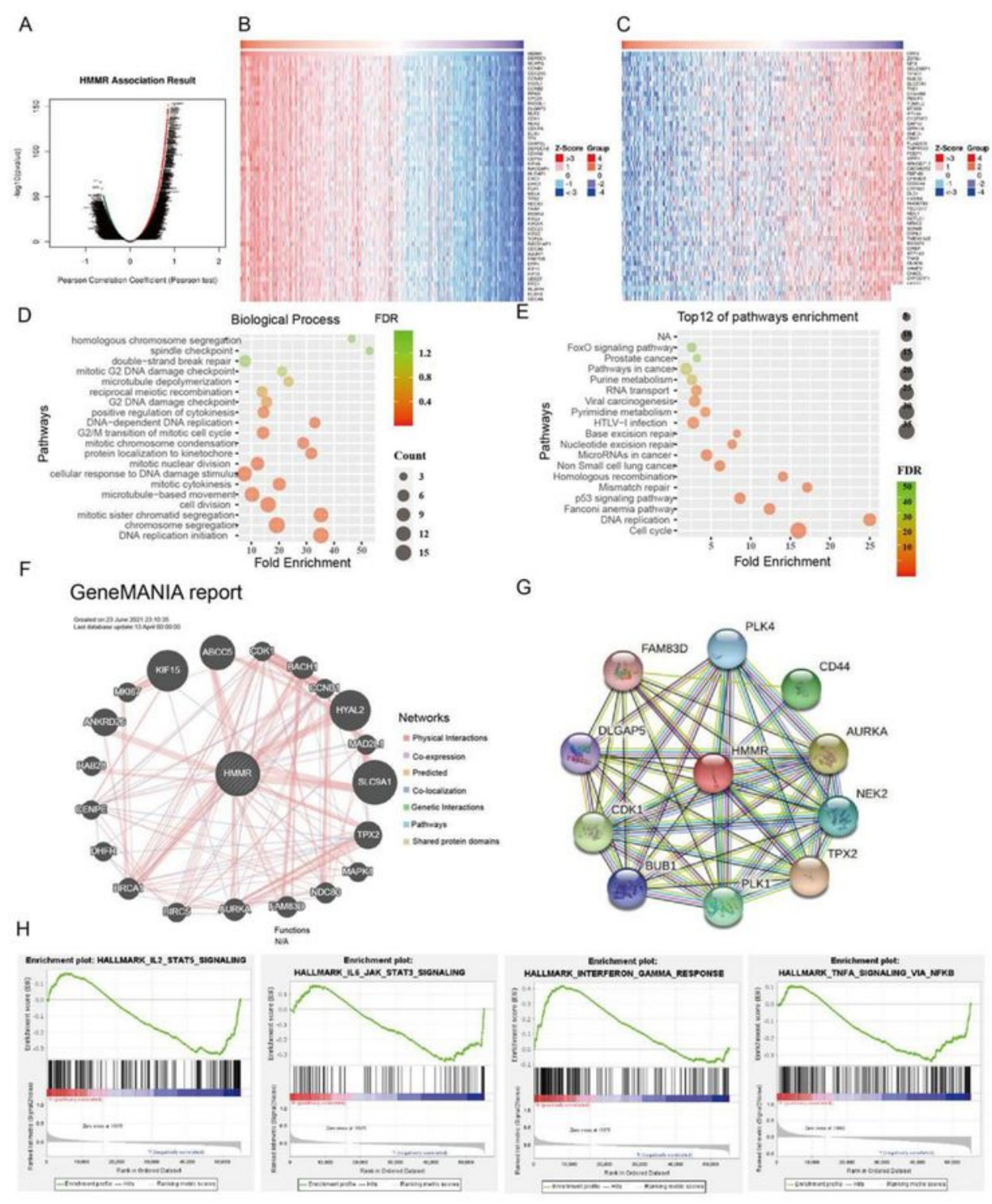

Figure 8 
KEGG Pathways Networks of HMMR in LUAD (a)The differentially expressed gene was related to display by Volcano plot (b-c) The positively and negatively related to h HMMR display by Heat maps (d) Analysis the Biology process of HMMR (e) Analysis the KEGG pathway of HMMR (f) Employed the GeneMania to construction the gene interaction network of HMMR (g) Employed the STRING to construction the protein interaction network of HMMR (h) The signaling pathway enriched employed the GSEA software.

A
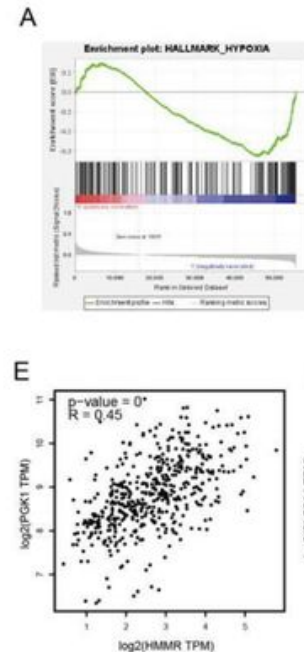

I

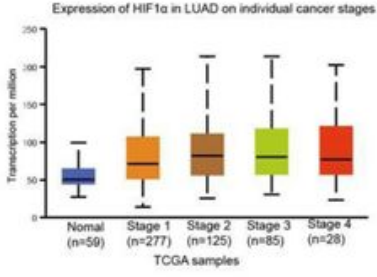

B
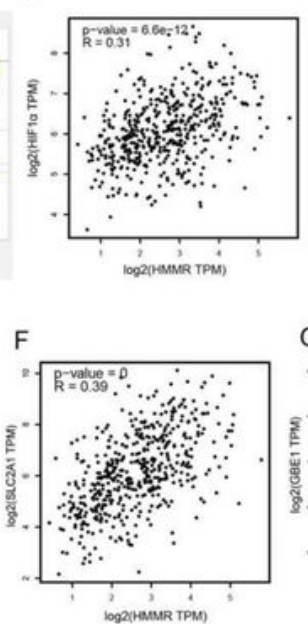

J

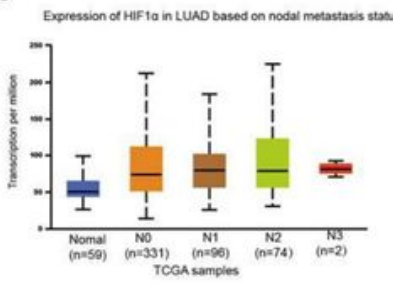

D
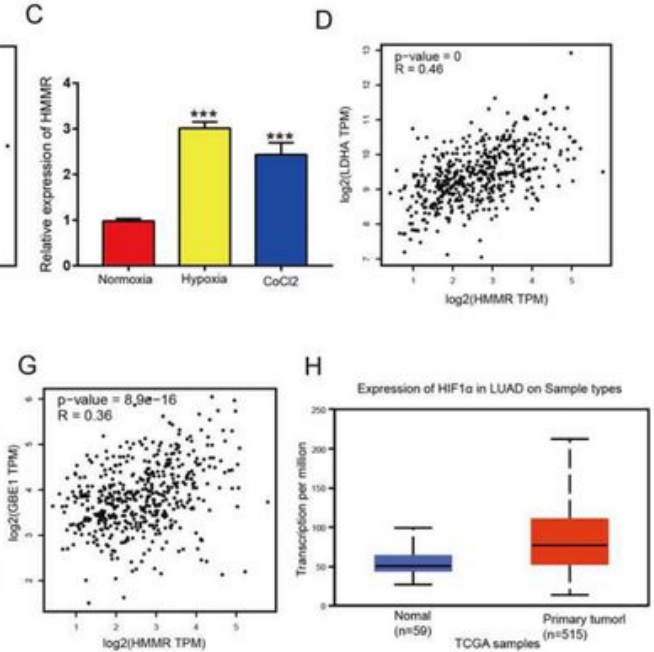

K

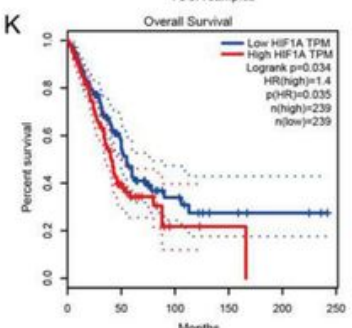

Figure 9 
HMMR is transcriptionally regulated by HIF-1a under hypoxia condition (a)The hypoxia pathway by GSEA analyses (b)The correlation between HIF-1a expression and HMMR expression in TCGA-LUAD (c) The expression of HMMR under different treat conditions (d) The correlation between HMMR expression and LDHA expression in TCGA-LUAD (e) The correlation between HMMR expression and PGK1 expression in TCGA-LUAD ( $f$ ) The correlation between HMMR expression and SLC2A1 expression in TCGA-LUAD ( $g$ ) The correlation between HMMR expression and GBE1 expression in TCGA-LUAD ( $h$ )The expression of HIF-1a in LUAD (i) The pathological stage analysis for HIF-1a in LUAD determined by UALCAN database (j) The lymph node metastatic stage analysis for HMMR in LUAD determined by UALCAN database $(k)$ The overall survival (OS) analysis for HIF-1ain LUAD determined by UALCAN database 


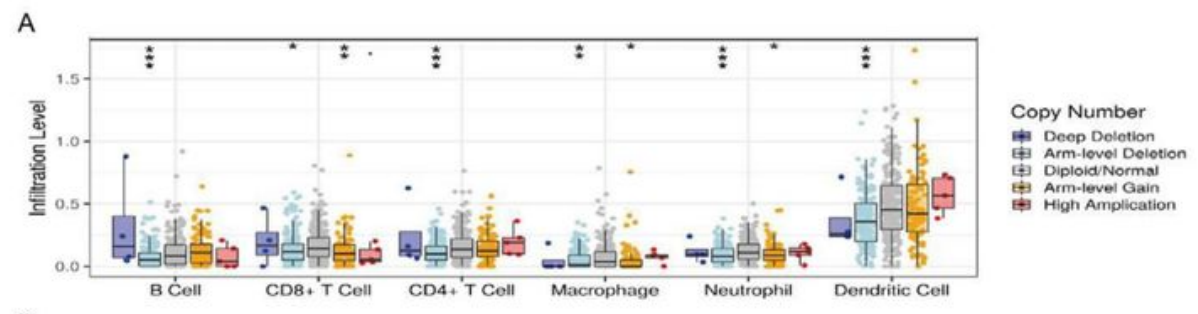

B
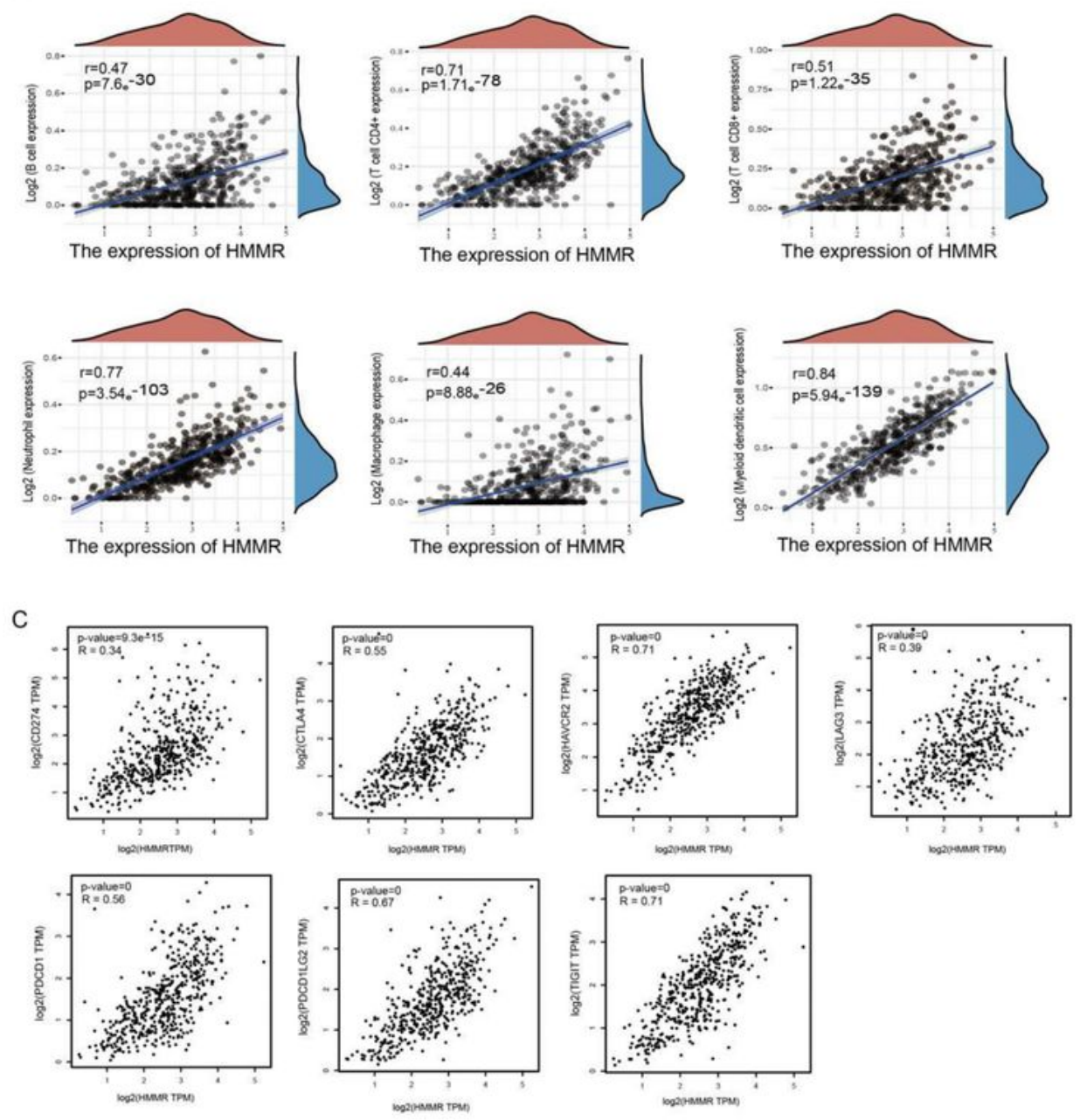

\section{Figure 10}

HMMR Expression was associated with Immune Infiltration in LUAD (a) The correlation between HMMR expression and immune cells in LUAD (b) The correlation between HMMR expression and immune infiltration in LUAD (c) The correlation between HMMR expression and immune checkpoints related gene in LUAD 

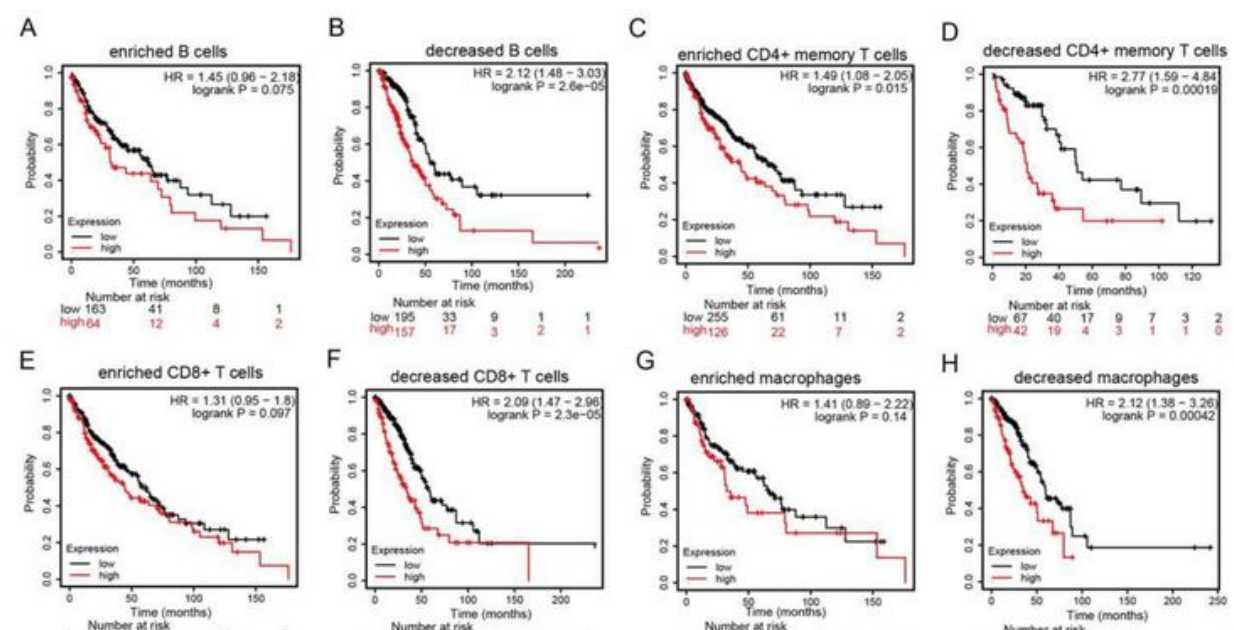

$\mathrm{F}$

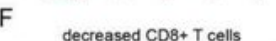

G
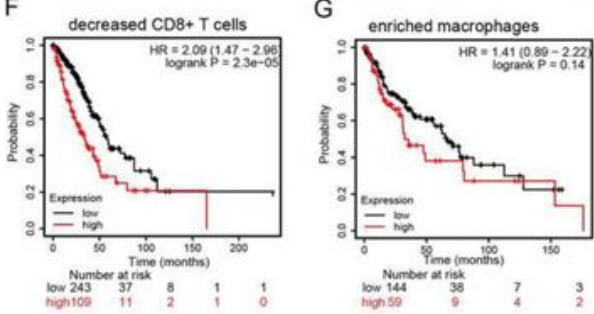

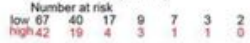

$\mathrm{H}$

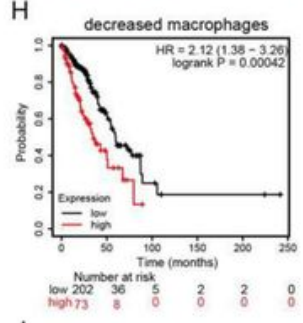

1 enciched natural viller $T$ cells
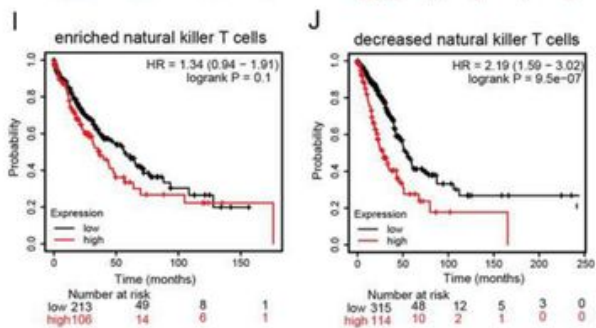

$J$

$\mathrm{K}$

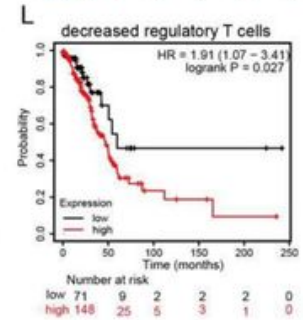

\section{Figure 11}

Prognostic Potential of HMMR Expressions in Different Tumors Based on Immune Cells (a-l) The relationship between the HMMR expression and OS based on immune cell subgroups emploted the KMplot $* p<0.05,{ }^{*} p<0.01, * \star * p<0.001$. 
A

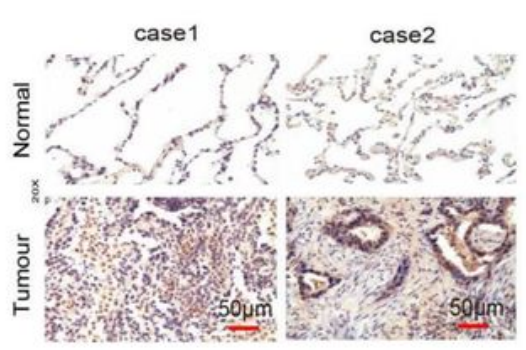

C

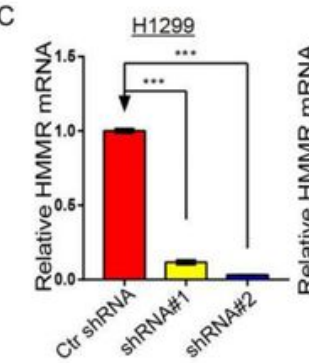

E

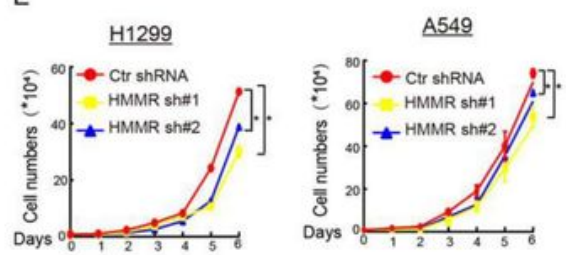

G

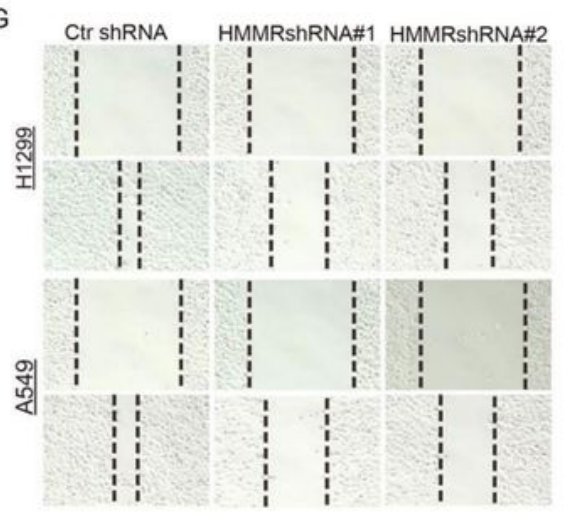

B

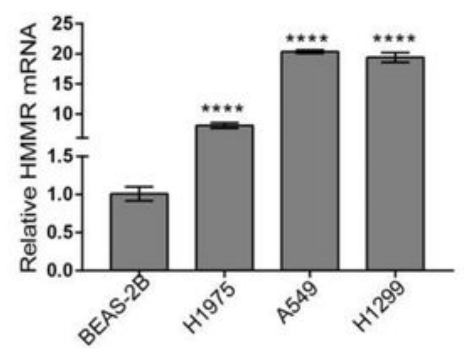

D
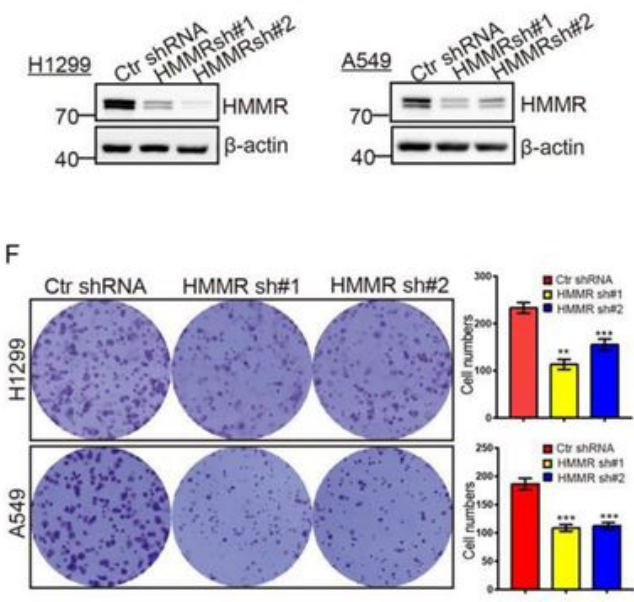

H Ctr ShRNA HMMR sh\#1 HMMR sh\#2
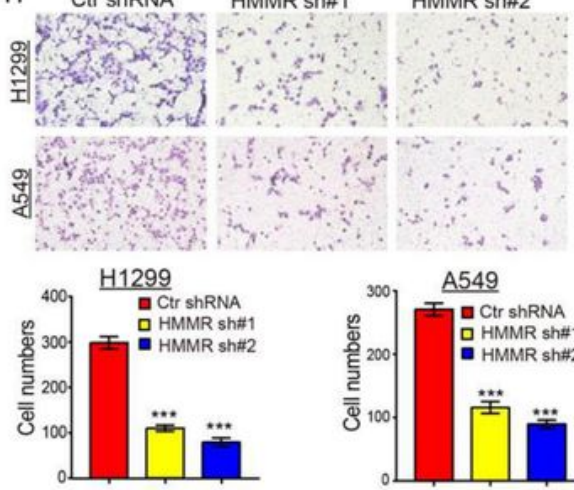

Figure 12

Depletion of HMMR inhibits growth and migration of NSCLC cells in vivro (a) IHC analysis of HMMR in LUAD. (b) The expression of HMMR in NSCLC cell lines by qRT-PCR (c-d) Establishment of HMMR knockdown in A549 cell lines and verified by qRT-PCR and Western blot (e) The growth curve assay was employed detect the proliferation of NSCLC cells. (f) The colony formation assay was employed detect 
the proliferation of NSCLC cells. (g) The transwell assay was employed detect the migartion of NSCLC cells. (h)The wound healing assay was employed detect the migartion of NSCLC cells. 